\title{
Fluid-Structure Interaction Effects on the Propulsion of an Flexible Composite Monofin
}

\author{
Adil El Baroudi ${ }^{1}$ and Fulgence Razafimahery ${ }^{2}$ \\ ${ }^{1}$ Arts et Métiers ParisTech, ENSAM Angers, 2 boulevard du Ronceray, 49035 Angers, France \\ ${ }^{2}$ IRMAR, Université de Rennes 1, Campus de Beaulieu, 35042 Rennes Cedex, France \\ Correspondence should be addressed to Fulgence Razafimahery; fulgence.razafimahery@univ-rennes1.fr
}

Received 17 May 2014; Revised 17 November 2014; Accepted 26 November 2014; Published 14 December 2014

Academic Editor: M’hamed Souli

Copyright (c) 2014 A. El Baroudi and F. Razafimahery. This is an open access article distributed under the Creative Commons Attribution License, which permits unrestricted use, distribution, and reproduction in any medium, provided the original work is properly cited.

\begin{abstract}
Finite element method has been used to analyze the propulsive efficiency of a swimming fin. Fluid-structure interaction model can be used to study the effects of added mass on the natural frequencies of a multilayer anisotropic fin oscillating in a compressible fluid. Water by neglecting viscidity effects has been considered as a surrounding fluid and the frequency response of the fin has been compared with that of vacuum conditions. It has been shown that because of the added mass effects in water environment, the natural frequencies of the fin decrease.
\end{abstract}

\section{Introduction}

Multilayer anisotropic structure has wide applications in areas such as modern construction engineering, biomechanical engineering, aerospace industries, aircraft construction, and the components of nuclear power plants. It is therefore very important that the modal and dynamic analysis of multilayer anisotropic structure when subjected to different loading conditions be clearly understood so that they may be safely used in these industrial applications.

It is well known that the natural frequencies of structures in contact with fluid are different from those in vacuum. Therefore, the prediction of natural frequency changes due to the presence of the fluid is important for designing structures which are in contact with or immersed in fluid. In general, the effect of the fluid force on the structure is represented as added mass, which lowers the natural frequency of the structure from that which would be measured in a vacuum. This decrease in the natural frequency of the fluid-structure system is caused by increasing the kinetic energy of the coupled system without a corresponding increase in strain energy.

In this paper the propulsive efficiency of a swimming fin has been studied. Dynamic analysis of aquatic locomotion is a fundamental parameter in the performance search. In the case of swimming with fins, the propulsive efficiency depends on several factors. Most models suggested aim to evaluate the dynamic performances, including drag and lift which are the two relevant parameters relevant to quantifing the propulsive efficiency of a fin. Some proposed models are essentially of discrete type $[1,2]$, while others, by being inspired by organs of propulsion of marine cetaceans, use continuous models $[3,4]$. Most of these authors do not account for the highly coupled nature of the problem. In fact, for rate of stresses observed in actual swimming, the coupling between the fluid and the fin becomes stronger.

\section{Governing Equations}

The numerical formulations used include the displacement formulation [5], the potential formulation [6], the pressure formulation [7], and the combination of some of them [8]. Finite element method is used to extract the natural frequencies and modal shapes. To compute the natural vibration modes of a fluid alone, the fluid is typically described either by pressure or by displacement potential variables. When the fluid is coupled with a solid, standard methods to solve (1) and (2) consist in eliminating either the pressure 


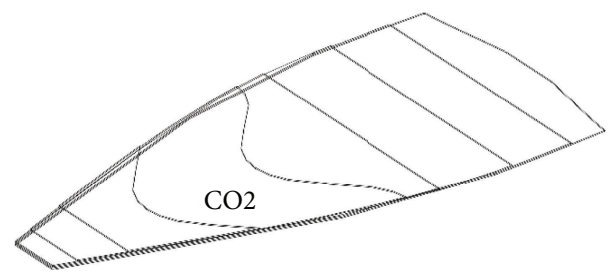

Figure 1: Geometry of 3D fin.

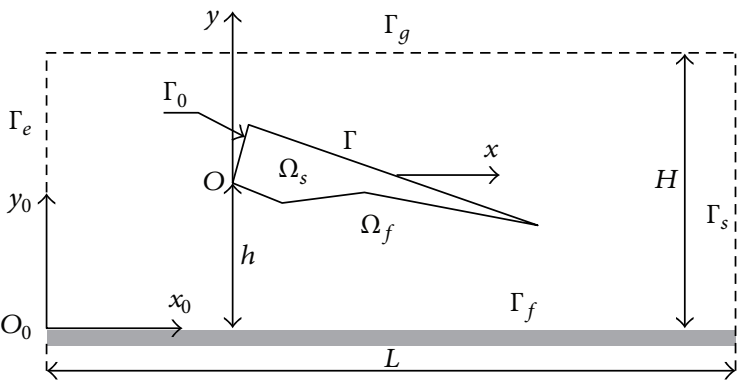

FIGURE 2: 2D computational domain.

(i) solid domain $\left(\Omega_{i}\right)$ :

$$
\begin{gathered}
\rho_{i} \frac{\partial^{2} \mathbf{u}_{i}}{\partial t^{2}}=\nabla \cdot \sigma\left(\mathbf{u}_{i}\right)+\rho_{i} \mathbf{F} \\
\sigma\left(\mathbf{u}_{i}\right)=\mathbb{K}\left(\theta_{i}\right) \varepsilon\left(\mathbf{u}_{i}\right)
\end{gathered}
$$

(ii) fluid domain $\left(\Omega_{f}\right)$ :

$$
\frac{1}{\rho_{0} c_{0}^{2}} \frac{\partial^{2} p}{\partial t^{2}}=\nabla \cdot\left[\frac{1}{\rho_{0}}\left(\nabla p-\rho_{0} \mathbf{F}\right)\right]
$$

(iii) fluid-solid interaction $(\Gamma)$ :

$$
\begin{gathered}
\sigma(\mathbf{u}) \mathbf{n}=-p \mathbf{n} \\
{\left[\nabla p-\rho_{0} \mathbf{F}\right] \cdot \mathbf{n}=-\rho_{0} \frac{\partial^{2} \mathbf{u}}{\partial t^{2}} \cdot \mathbf{n}}
\end{gathered}
$$

(iv) other boundary conditions:

$$
\begin{gathered}
\mathbf{u}=\mathbf{0} \quad\left(\Gamma_{0}\right), \\
{\left[\nabla p-\rho_{0} \mathbf{F}\right] \cdot \mathbf{n}=0 \quad\left(\Gamma_{f}\right),} \\
p=0 \quad\left(\Gamma_{e} \cup \Gamma_{g} \cup \Gamma_{s}\right) .
\end{gathered}
$$

\section{Modal Analysis of Composite Monofin}

Modal analysis of elastic submerged structures is needed in every modern construction and has wide engineering application especially in ocean engineering. In this study, modal analysis is important to predict the dynamic behavior of the submerged fin. It is well known that the natural frequencies of the submerged elastic structures are different from those in vacuum. The effect of fluid forces on the submerged fin is represented as added mass, which decreases the natural frequencies of the submerged fin from those which would be measured in the vacuum. This decrease in the natural frequencies of the submerged structures is caused by the increase of the kinetic energy of the fluid-fin system without a corresponding increase in strain energy. This step seems important to calculate the variations of natural frequencies of the fin for different situations. For this, we looked at the modes of the fin in the vacuum and water. to the fin, the problem is to find $\left(\mathbf{u}_{i}, p\right)$ solutions: 
Indeed, to test the quality of a fin, it is usual to search its quasi-static deformed shape and dynamic response in air. The aim is to check if results of tests carried out of the water are strongly influenced by the presence of the surrounding fluid. In addition, frequencies can have accurate information in the dynamic behavior of the system. By introducing the spaces of test functions $\mathbf{V}=\left\{\mathbf{v} \in \mathbf{H}^{1}\left(\Omega_{s}\right), \mathbf{v}=\mathbf{0}\left(\Gamma_{0}\right)\right\}$ and $\phi \in Q=H^{1}\left(\Omega_{f}\right)$, the weak formulation of (1) and (2) holds:

$$
\begin{gathered}
\int_{\Omega_{s}} \sigma(\mathbf{u}): \varepsilon(\mathbf{v}) d x-\omega^{2} \int_{\Omega_{s}} \rho \mathbf{u} \cdot \mathbf{v} d x \\
\quad+\int_{\Gamma} p \mathbf{v} \cdot \mathbf{n} d \Gamma=0, \\
\int_{\Omega_{f}} \frac{1}{\rho_{0}} \nabla p \cdot \nabla \phi d x-\omega^{2} \int_{\Omega_{f}} \frac{p \phi}{\rho_{0} c_{0}^{2}} d x \\
-\omega^{2} \int_{\Gamma} \mathbf{u} \cdot \mathbf{n} \phi d \Gamma=0,
\end{gathered}
$$

where

$$
\begin{aligned}
\int_{\Omega_{s}} \sigma(\mathbf{u}): \varepsilon(\mathbf{v}) d x & =\sum_{i=1}^{N_{L}} \int_{\Omega_{i}} \sigma\left(\mathbf{u}_{i}\right): \varepsilon\left(\mathbf{v}_{i}\right) d x \\
\int_{\Omega_{s}} \rho \mathbf{u} \cdot \mathbf{v} d x & =\sum_{i=1}^{N_{L}} \int_{\Omega_{i}} \rho_{i} \mathbf{u}_{i} \cdot \mathbf{v}_{i} d x
\end{aligned}
$$

$N_{L}$ is a number of layers. Using Lagrange finite elements, where $\mathbf{u}_{h} \in \mathbb{P}_{2} \times \mathbb{P}_{2}$ and $p_{h} \in \mathbb{P}_{1}$, discretization of the weak formulation (5) induces a nonsymmetrical system:

$$
\left[\begin{array}{cc}
\mathbb{K}_{s} & \mathbb{B} \\
\mathbb{O} & \mathbb{K}_{p}
\end{array}\right]\left[\begin{array}{l}
\mathbf{U} \\
\mathbf{P}
\end{array}\right]=\omega^{2}\left[\begin{array}{cc}
\mathbb{M}_{s} & \mathbb{O} \\
\mathbb{M}_{a} & \mathbb{M}_{p}
\end{array}\right]\left[\begin{array}{l}
\mathbf{U} \\
\mathbf{P}
\end{array}\right]
$$

where $\mathbf{U}$ and $\mathbf{P}$ are the vectors of nodal values for $\mathbf{u}$ and $p$, respectively. The submatrices of (7) are defined by

$$
\begin{aligned}
\mathbf{V}^{T} \mathbb{K}_{s} \mathbf{U} & =\int_{\Omega_{s}} \sigma(\mathbf{u}): \varepsilon(\mathbf{v}) d x, \\
\Phi^{T} \mathbb{K}_{p} \mathbf{P} & =\int_{\Omega_{f}} \frac{p \phi}{c_{0}^{2}} d x, \\
\mathbf{V}^{T} \mathbb{M}_{s} \mathbf{U} & =\int_{\Omega_{s}} \rho \mathbf{u} \cdot \mathbf{v} d x, \\
\mathbf{V}^{T} \mathbb{B} \mathbf{P} & =\int_{\Gamma} p \mathbf{v} \cdot \mathbf{n} d \Gamma, \\
\Phi^{T} \mathbb{M}_{p} \mathbf{P} & =\int_{\Omega_{f}} \nabla p \cdot \nabla \phi d x, \\
\Phi^{T} \mathbb{M}_{a} \mathbf{U} & =\int_{\Gamma} \rho_{0} \mathbf{u} \cdot \mathbf{n} \phi d \Gamma,
\end{aligned}
$$

where $\mathbf{V}$ and $\Phi$ are the vectors of nodal values for $\mathbf{v}$ and $\phi$, respectively. $\mathbb{M}_{a}$ is the added mass matrix (symmetric and positive definite [11]). The nonsymmetric system (7) was solved using the commercial software Comsol Multiphysics.
TABLE 1: Natural frequencies $f(\mathrm{~Hz})$ of the fin in the case of $0^{\circ} / 90^{\circ} / 0^{\circ} / 90^{\circ} / 0^{\circ}$.

\begin{tabular}{lccc}
\hline Oriented fibers & \multicolumn{3}{c}{ In vacuum } \\
& $f_{1}$ & $f_{2}$ & $f_{3}$ \\
\hline $0^{\circ} / 90^{\circ}$ & 12.6 & 76.0 & 205.3 \\
$0^{\circ} / 90^{\circ} / 0^{\circ}$ & 29.3 & 122.9 & 265.0 \\
$0^{\circ} / 90^{\circ} / 0^{\circ} / 90^{\circ}$ & 22.6 & 90.1 & 192.0 \\
$0^{\circ} / 90^{\circ} / 0^{\circ} / 90^{\circ} / 0^{\circ}$ & 28.8 & 79.7 & 185.1 \\
\hline
\end{tabular}

TABLE 2: Natural frequencies $f(\mathrm{~Hz})$ of the fin in the case of $0^{\circ} / 90^{\circ} / 0^{\circ} / 90^{\circ} / 0^{\circ}$.

\begin{tabular}{lccc}
\hline Oriented fibers & \multicolumn{3}{c}{ In water } \\
& $f_{1}$ & $f_{2}$ & $f_{3}$ \\
\hline $0^{\circ} / 90^{\circ}$ & 1.7 & 46.0 & 103.1 \\
$0^{\circ} / 90^{\circ} / 0^{\circ}$ & 3.5 & 21.7 & 55.1 \\
$0^{\circ} / 90^{\circ} / 0^{\circ} / 90^{\circ}$ & 2.4 & 14.5 & 37.3 \\
$0^{\circ} / 90^{\circ} / 0^{\circ} / 90^{\circ} / 0^{\circ}$ & 2.7 & 11.8 & 33.4 \\
\hline
\end{tabular}

TABLE 3: Natural frequencies $f(\mathrm{~Hz})$ of the fin in the case of $90^{\circ} / 0^{\circ} / 90^{\circ} / 0^{\circ} / 90^{\circ}$.

\begin{tabular}{lccc}
\hline Oriented fibers & \multicolumn{3}{c}{ In vacuum } \\
& $f_{1}$ & $f_{2}$ & $f_{3}$ \\
\hline $90^{\circ} / 0^{\circ}$ & 12.6 & 73.5 & 158.4 \\
$90^{\circ} / 0^{\circ} / 90^{\circ}$ & 9.5 & 51.3 & 105.5 \\
$90^{\circ} / 0^{\circ} / 90^{\circ} / 0^{\circ}$ & 22.1 & 56.2 & 115.5 \\
$90^{\circ} / 0^{\circ} / 90^{\circ} / 0^{\circ} / 90^{\circ}$ & 17.4 & 44.8 & 91.6 \\
\hline
\end{tabular}

TABLE 4: Natural frequencies $f(\mathrm{~Hz})$ of the fin in the case of $90^{\circ} / 0^{\circ} / 90^{\circ} / 0^{\circ} / 90^{\circ}$.

\begin{tabular}{lccc}
\hline Oriented fibers & \multicolumn{3}{c}{ In a water } \\
& $f_{1}$ & $f_{2}$ & $f_{3}$ \\
\hline $90^{\circ} / 0^{\circ}$ & 1.7 & 13.3 & 39.4 \\
$90^{\circ} / 0^{\circ} / 90^{\circ}$ & 1.1 & 8.7 & 23.8 \\
$90^{\circ} / 0^{\circ} / 90^{\circ} / 0^{\circ}$ & 2.2 & 9.5 & 23.8 \\
$90^{\circ} / 0^{\circ} / 90^{\circ} / 0^{\circ} / 90^{\circ}$ & 1.6 & 6.8 & 17.2 \\
\hline
\end{tabular}

Two types of calculations were carried out. The first is when the palm is plunged into the vacuum and the second into water. We give below the results for a model of up to five layers $\left(N_{L}=5\right)$ and the natural frequencies in vacuum and water. The fibers of each layer are arranged alternately along the two directions orthogonal axes $\mathbf{x}$ and $\mathbf{y}$ of the mean plane of the fin. Tables 2 and 4 show that arrangement of layers has a strong influence on the natural frequencies, and the added mass decreases the natural frequencies. Figures 3 and 4 show that arrangement of layers has no influence on the coupled modal shapes (Tables 1 and 3 ).

\section{Dynamic Analysis of Composite Monofin}

The dynamic problem was conducted using the data proposed in [14]. For this, the fin is subjected to combined 


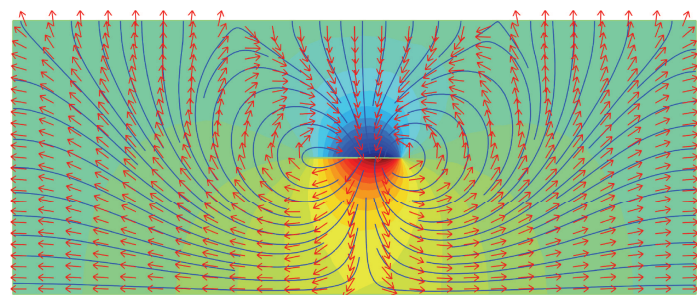

(a) Coupled mode $1\left(f_{1}=2.7(\mathrm{~Hz})\right)$

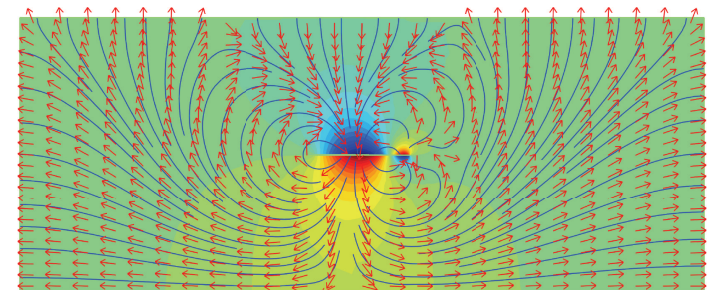

(b) Coupled mode $2\left(f_{2}=11.8(\mathrm{~Hz})\right)$

Figure 3: The colors pertain to the pressure field and the arrows to the velocity field in the case of $0^{\circ} / 90^{\circ} / 0^{\circ} / 90^{\circ} / 0^{\circ}$.

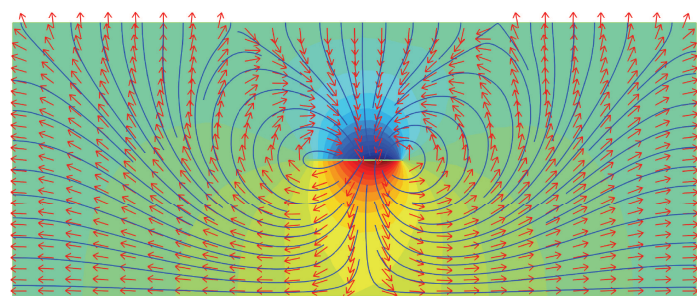

(a) Coupled mode $1\left(f_{1}=1.6(\mathrm{~Hz})\right)$

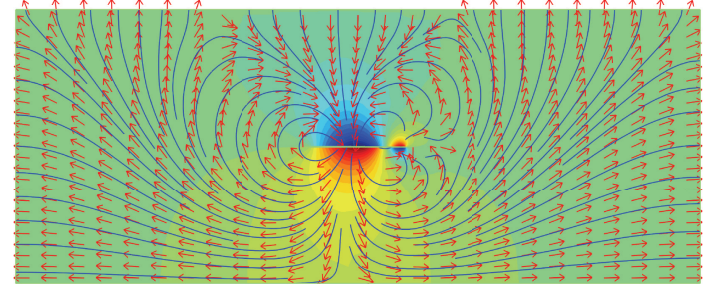

(b) Coupled mode $2\left(f_{2}=6.8(\mathrm{~Hz})\right)$

FIGURE 4: The colors pertain to the pressure field and the arrows to the velocity field in the case of $90^{\circ} / 0^{\circ} / 90^{\circ} / 0^{\circ} / 90^{\circ}$.

translational and rotation motions. In this case, the quantity F introduced in the model problem (1) has the expression

$$
\mathbf{F}=\left\{\begin{array}{l}
x \dot{\omega}^{2}(t)+y \ddot{\omega}(t)-\ddot{h}(t) \sin [\omega(t)] \\
y \dot{\omega}^{2}(t)-x \ddot{\omega}(t)-\ddot{h}(t) \cos [\omega(t)]
\end{array}\right\}
$$

where

$$
\begin{aligned}
& \omega(t)=\theta_{0} \sin (2 \pi f t), \\
& h(t)=h_{0} \sin (2 \pi f t-\psi),
\end{aligned}
$$

$\theta_{0}=40^{\circ}, \psi=\pi / 2, h_{0}=1 c, f=0.225[\mathrm{~Hz}]$, and $c=0.7$ is the chord of the profile, that is to say, the length of the fin. The phase $\psi$ is introduced to model the muscle dissymmetry.

To avoid a resonant frequency, the excitation frequency is taken far enough from the first natural frequency of the coupled system. The hydrodynamic parameters that seem most relevant are the total force $\mathbf{R}\left(=\int_{\Gamma} \sigma(\mathbf{u}) \mathbf{n} d \Gamma\right)$ exerted on the fin during the movement phase. The two components of $\mathbf{R}$ are, respectively, the drag $(D)$ and lift $(L)$ of the fin. The quantity $T=-D$ is called thrust. Different types of layers exist in the manufacture of fins. Throughout the model the thickness of the fin is fixed in advance. We use the same physical characteristics as in the case of modal analysis.
Using the same notation as before, the weak formulation of boundary value problem (1) and (2) is then written:

$$
\begin{aligned}
& \frac{d^{2}}{d t^{2}} \int_{\Omega_{s}} \rho \mathbf{u} \cdot \mathbf{v} d x+\int_{\Omega_{s}} \sigma(\mathbf{u}): \varepsilon(\mathbf{v}) d x \\
& \quad+\int_{\Gamma} p \mathbf{v} \cdot \mathbf{n} d \Gamma=-\int_{\Omega_{s}} \rho \mathbf{F} \cdot \mathbf{v} d x, \\
& \frac{d^{2}}{d t^{2}}\left(\int_{\Omega_{f}} \frac{p \phi}{c_{0}^{2}} d x+\int_{\Gamma} \rho_{0} \mathbf{u} \cdot \mathbf{n} \phi d \Gamma\right) \\
& \quad+\int_{\Omega_{f}} \nabla p \cdot \nabla \phi d x=\int_{\Omega_{f}} \rho_{0} \mathbf{F} \cdot \nabla \phi d x .
\end{aligned}
$$

In this section, we use a particular kinematics proposed in $[14,15]$, even if our models are not exactly similar. Indeed, the kinematics will allow us in the future to develop a new experimental protocol for measuring various hydrodynamic parameters of a fin. As the model problem (1)-(2) is linear, it is interesting to see the different contributions of each elementary movement in the dynamic response of the fin.

4.1. Dynamic Response in the Case of Translational Motion. The rotation $\omega(t)$ is canceled and the movement is then sinusoidal along the direction $y$. Figure 5 shows that the two-layer model seems to give a greater thrust than the other models. This is consistent with the results of modal analysis, where this is the first model that has the lowest frequency. This type of movement is not interesting for the propulsive efficiency. Indeed, it leads to a zero mean propulsive efficiency. On the other hand, we see a greater amplitude for thrust, compared to the lift. 


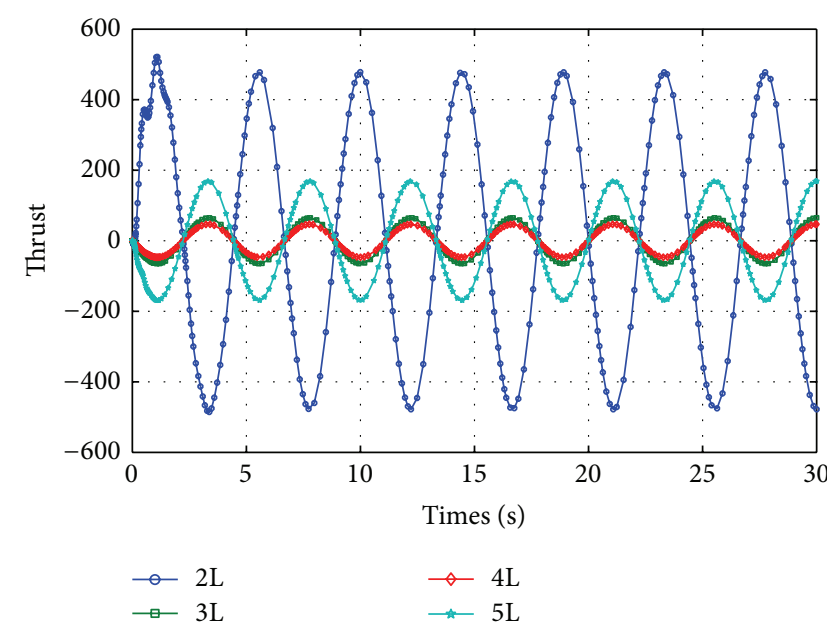

(a) $0^{\circ} / 90^{\circ} / 0^{\circ} / 90^{\circ} / 0^{\circ}$

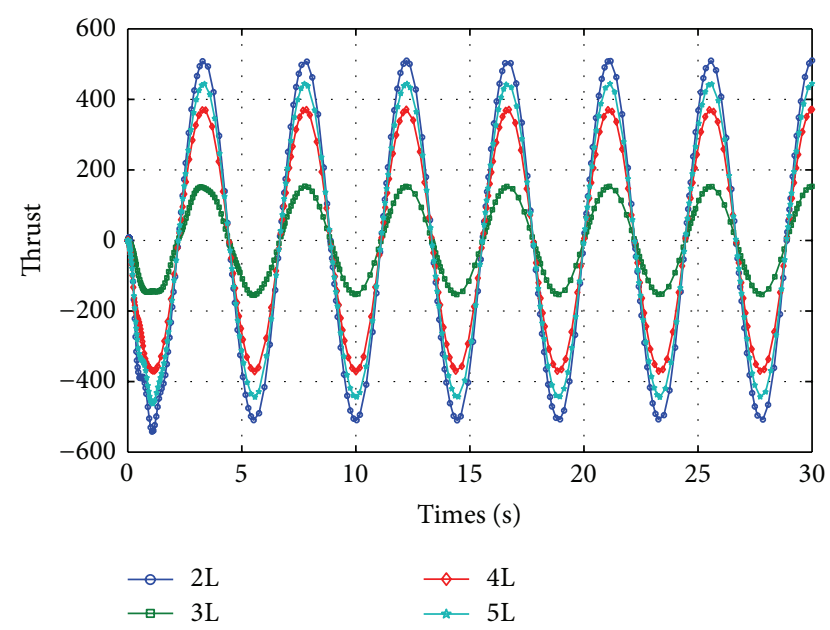

(b) $90^{\circ} / 0^{\circ} / 90^{\circ} / 0^{\circ} / 90^{\circ}$

Figure 5: Thrust of fin in the case of translation motion.

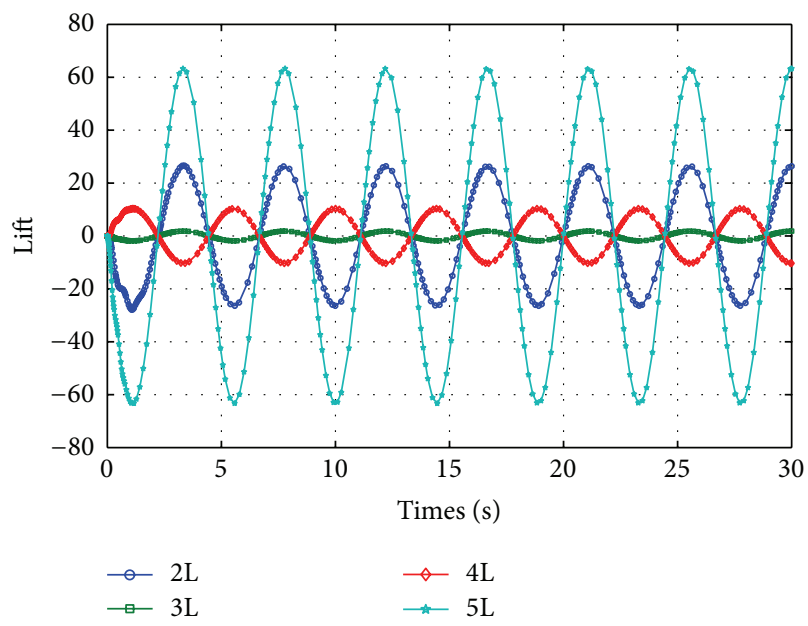

(a) $0^{\circ} / 90^{\circ} / 0^{\circ} / 90^{\circ} / 0^{\circ}$

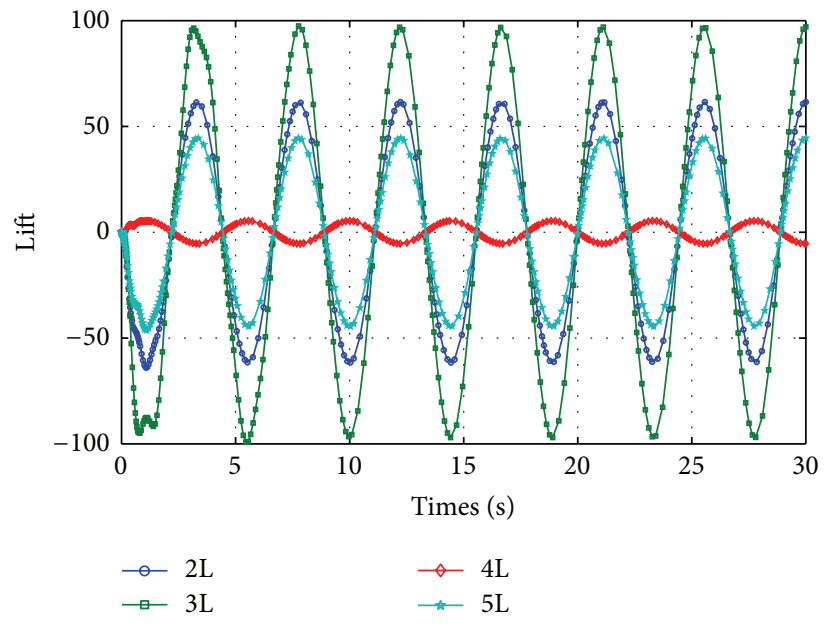

(b) $90^{\circ} / 0^{\circ} / 90^{\circ} / 0^{\circ} / 90^{\circ}$

FIGURE 6: Lift of fin in the case of translation motion.

4.2. Dynamic Response in the Case of Rotation Motion. The function $h(t)$ is canceled and the movement will be a sinusoidal rotation around foot. According to Figure 7, the two-layer model always gives a greater thrust than the other models. But by eliminating this model in the response curves, we can see that the five-layer model gives the best performance. The three-layer model gives a better lift compared to other models. Thus, this type of movement provides a propulsive efficiency rather interesting. This phenomenon is also well observed in the movement of marine mammals. On the other hand, such movement can be interesting if you want to stay stationary at one position.

4.3. Dynamic Response in the Case of Combined RotationalTranslational Motion. In order to have a reasonable performance of the system, we must combine both translational and rotation motion and take the full expression of the excitation force F. According to Figure 9, the two-layer model always gives a greater thrust than the other models. In general, the three-layer model seems to give a better compromise. Indeed, its thrust remains positive all the time, while its lift is of negative value and has less importance than other models. It is possible that by varying some physical parameters, we can significantly reduce hydrodynamic quantities, such as the moment and lift.

\section{Conclusions}

Finally, the above results allow us to draw some conclusions.

(i) The presence of layers provides some flexibility as indicated by the results of modal analysis. The first mode is flexural type, which justifies the use of models proposed in [1].

(ii) Fins with anisotropic material structures allow implementing a technique of layers parameterization to 


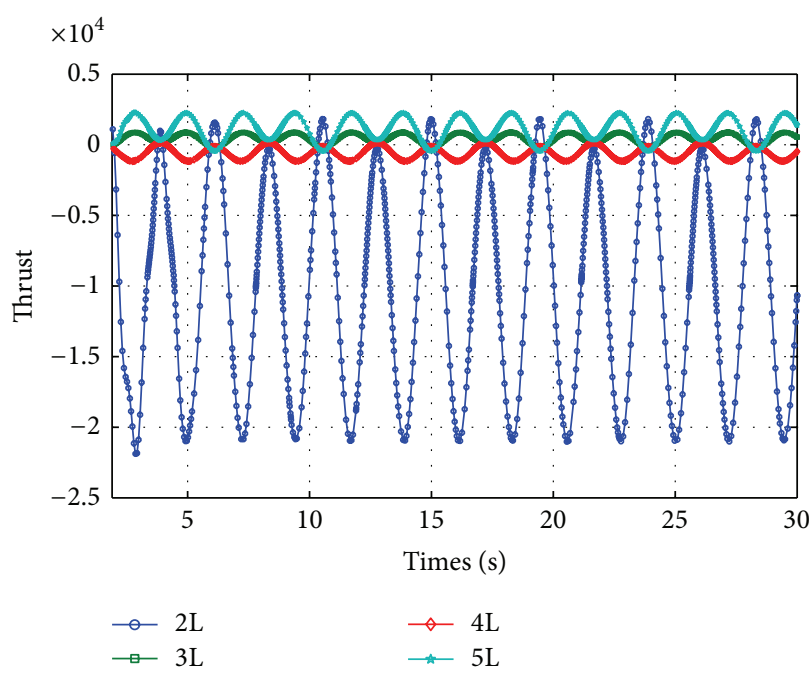

(a) $0^{\circ} / 90^{\circ} / 0^{\circ} / 90^{\circ} / 0^{\circ}$

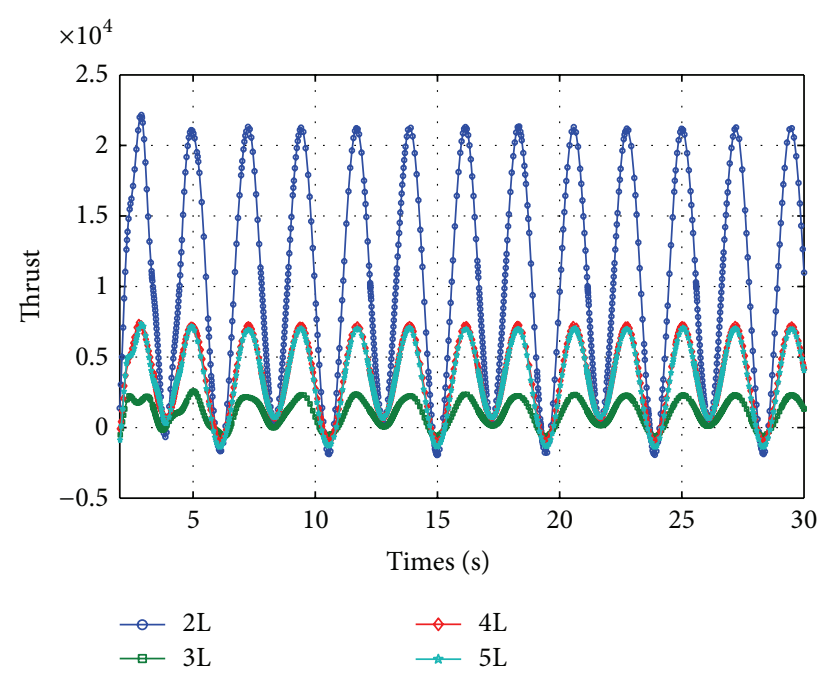

(b) $90^{\circ} / 0^{\circ} / 90^{\circ} / 0^{\circ} / 90^{\circ}$

Figure 7: Thrust of fin in the case of rotation motion.

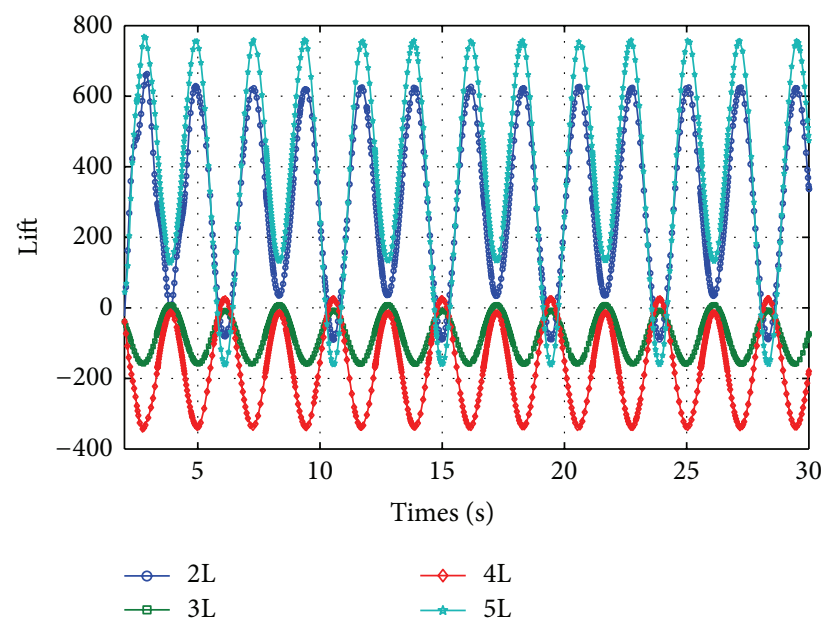

(a) $0^{\circ} / 90^{\circ} / 0^{\circ} / 90^{\circ} / 0^{\circ}$

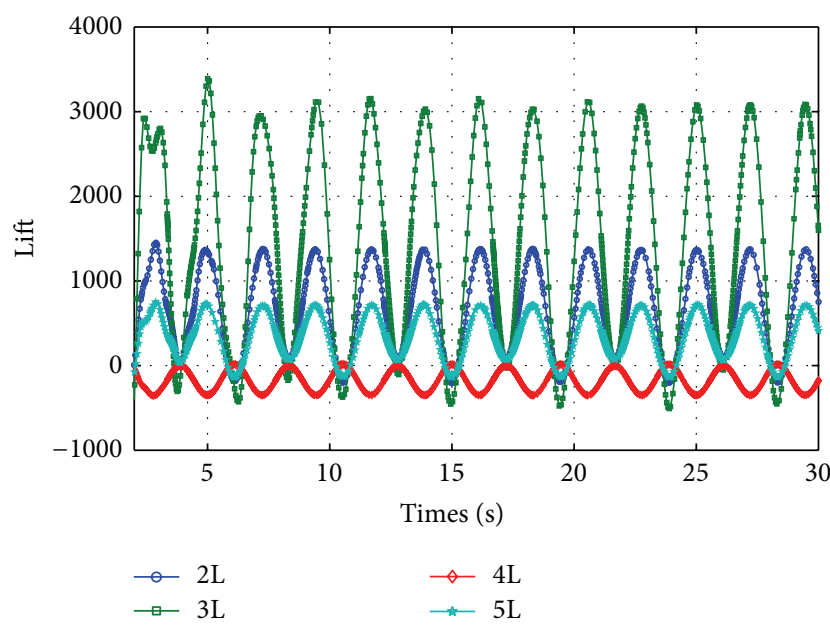

(b) $90^{\circ} / 0^{\circ} / 90^{\circ} / 0^{\circ} / 90^{\circ}$

FIGURE 8: Lift of fin in the case of rotation motion.

improve performance. It is quite possible now to bring special attention to the structure of the layers and types of constituent materials thereof.

(iii) The sensitivity of the dynamic behavior of the model with respect to the materials used and the boundary conditions for the fluid domain should be noted. Indeed, the presence or absence of rigid walls alters significantly the natural modes of the coupled system. Thus, the dynamic behavior of a swimmer depends on the localization in the pool where it is at the given moment. To obtain a better thrust, the fin has to be elastic and has to be sought at least in rotation. The amplitude of the vertical translation must be controlled to avoid a too high lift, in order to remain at a constant depth. The use of multilayer fins allows controlling an excessive variation of lift (Figures 6, 8, and 10).

(iv) Most experimental results we know $[16,17]$ are mainly interested in kinematic aspect of the mechanical problem. Nevertheless, the results obtained in the case of a rigid fin [14] allowed us to have a basis for comparison. We found that dynamic responses curves are similar but with different amplitudes. These differences in results obtained can be explained by the type of models used (rigid fin and flexible composite fin).

In this paper the modal and dynamic analysis is proposed to understand the behavior of a flexible composite fin with a good accuracy. The publications in the literatures deal with the behavior of fins; few authors have not studied the case of coupled boundary conditions [18]. It is for this reason this 


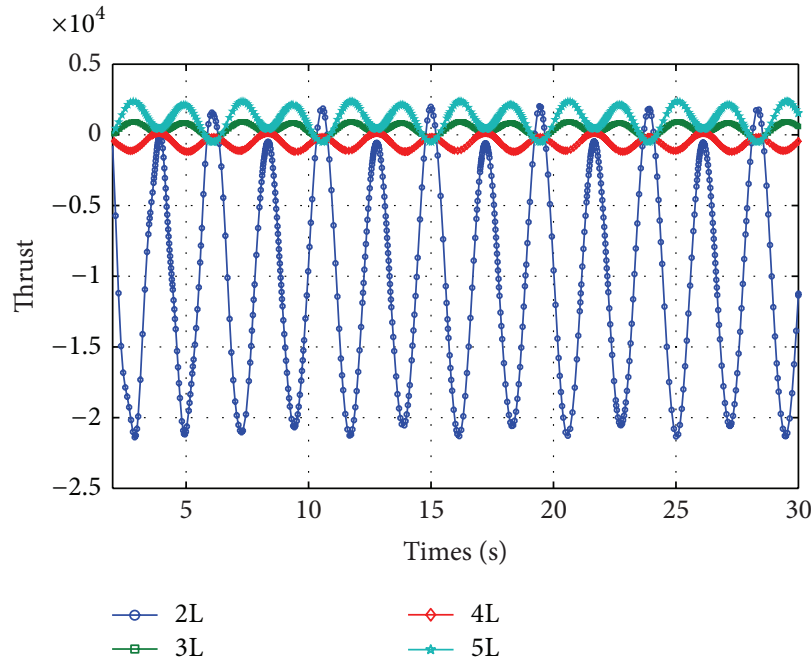

(a) $0^{\circ} / 90^{\circ} / 0^{\circ} / 90^{\circ} / 0^{\circ}$

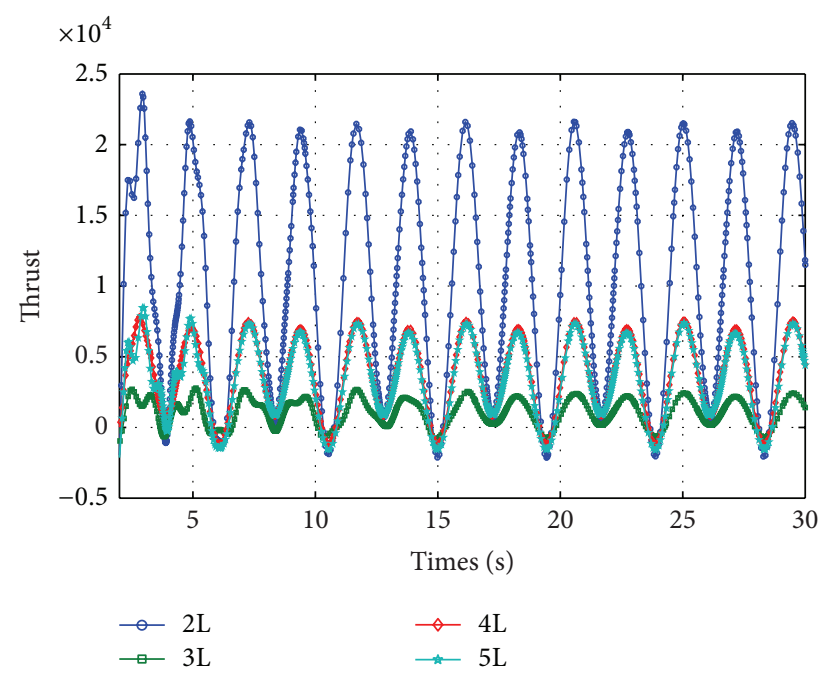

(b) $90^{\circ} / 0^{\circ} / 90^{\circ} / 0^{\circ} / 90^{\circ}$

FIgURE 9: Thrust of fin in the case of combined rotational and translational motion.

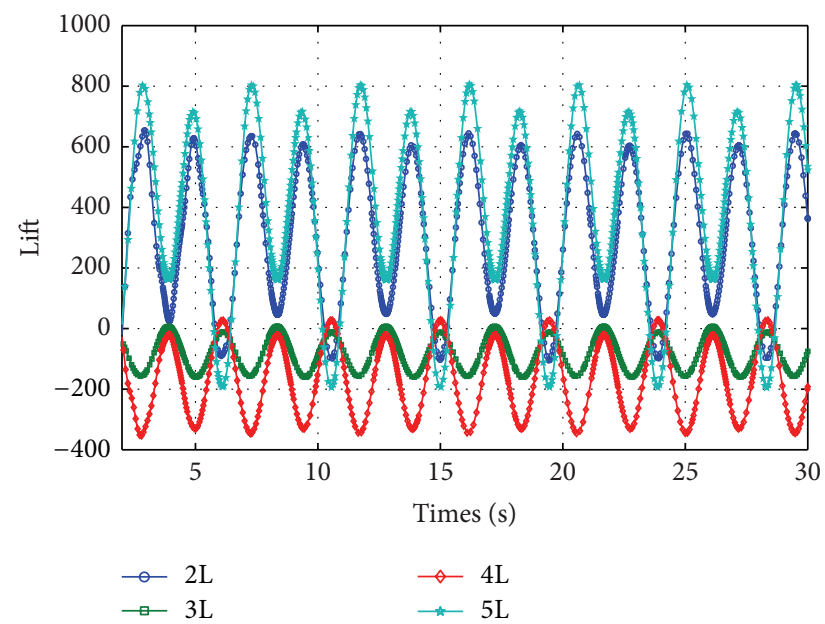

(a) $0^{\circ} / 90^{\circ} / 0^{\circ} / 90^{\circ} / 0^{\circ}$

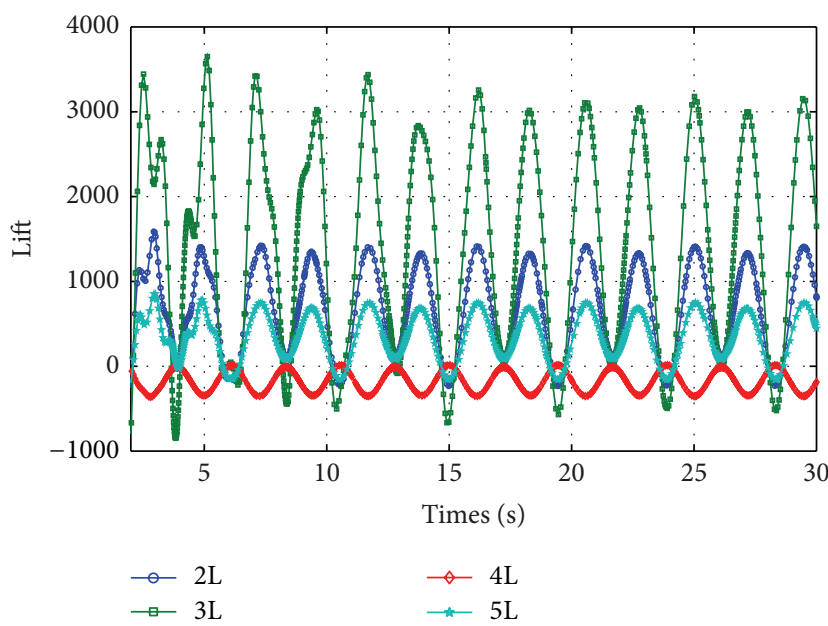

(b) $90^{\circ} / 0^{\circ} / 90^{\circ} / 0^{\circ} / 90^{\circ}$

FIGURE 10: Lift of fin in the case of combined rotational and translational motion.

study was conducted on the request of a company specializing in the design of fins in order to improve the propulsion of a flexible composite fin.

\section{Conflict of Interests}

The authors declare that there is no conflict of interests regarding the publication of this paper.

\section{References}

[1] J. C. Mollendorf, J. D. Felske, S. Samimy, and D. R. Pendergast, "A fluid/solid model for predicting slender body deflection in a moving fluid," Journal of Applied Mechanics, Transactions ASME, vol. 70, no. 3, pp. 346-350, 2003.

[2] M. A. Luersen, R. Le Riche, D. Lemosse, and O. Le Maître, "A computationally efficient approach to swimming monofin optimization," Structural and Multidisciplinary Optimization, vol. 31, no. 6, pp. 488-496, 2006.

[3] I. Akhtar, R. Mittal, G. V. Lauder, and E. Drucker, "Hydrodynamics of a biologically inspired tandem flapping foil configuration," Theoretical and Computational Fluid Dynamics, vol. 21, no. 3, pp. 155-170, 2007.

[4] Y. Yadykin, V. Tenetov, and D. Levin, "The added mass of a flexible plate oscillating in a fluid," Journal of Fluids and Structures, vol. 17, no. 1, pp. 115-123, 2003.

[5] M. A. Hamdi, Y. Ousset, and G. Verchery, "A displacement method for analysis of vibrations of coupled fluid-structure systems," International Journal for Numerical Methods in Engineering, vol. 13, no. 1, pp. 139-150, 1978.

[6] H. Morand and R. Ohayon, "Substructure variational analysis of the vibrations of coupled fluid-structure systems: finite element results," International Journal for Numerical Methods in Engineering, vol. 14, no. 5, pp. 741-755, 1979. 
[7] A. A. Parthasarathi, K. Grosh, and A. L. Nuttall, "Threedimensional numerical modeling for global cochlear dynamics," Journal of the Acoustical Society of America, vol. 107, no. 1, pp. 474-485, 2000.

[8] K. J. Bathe, Finite Element Procedures, Prentice Hall, Englewood Cliffs, NJ, USA, 1996.

[9] M. Mellado and R. Rodriguez, "Efficient solution of fluidstructure vibration problems," Applied Numerical Mathematics, vol. 36, no. 4, pp. 389-400, 2001.

[10] O. C. Zienkiewich and R. L. Taylor, The Finite Element Method, vol. 2, McGraw-Hill, 1989.

[11] H. J.-P. Morand and R. Ohayon, Fluid Structure Interaction, John Wiley \& Sons, 1995.

[12] A. El Baroudi, Modeling in fluid-structure-interaction: applications to the problems resulting from biomechanics [Ph.D. thesis], Université of Rennes 1, 2010.

[13] F. Axisa and J. Antunes, Modeling of Mechanical Systems: Fluid Structure Interaction, vol. 3, Elsevier, 2007.

[14] D. A. Read, F. S. Hover, and M. S. Triantafyllou, "Forces on oscillating foils for propulsion and maneuvering," Journal of Fluids and Structures, vol. 17, no. 1, pp. 163-183, 2003.

[15] S. Shin, S. Y. Bae, I. C. Kim, and Y. J. Kim, "Effects of flexibility on propulsive force acting on a heaving foil," Ocean Engineering, vol. 36, no. 3-4, pp. 285-294, 2009.

[16] G. Nicolas and B. Bideau, "A kinematic and dynamic comparison of surface and underwater displacement in high level monofin swimming," Human Movement Science, vol. 28, no. 4, pp. 480-493, 2009.

[17] G. Nicolas, B. Bideau, N. Bideau, B. Colobert, G. Le Guerroue, and P. Delamarche, "A new system for analyzing swim fin propulsion based on human kinematic data," Journal of Biomechanics, vol. 43, no. 10, pp. 1884-1889, 2010.

[18] M. A. Luersen and R. Le Riche, "Adapting ply drop positions for compensating fabric changes-application to swimming monofins," Finite Elements in Analysis and Design, vol. 46, no. 10, pp. 930-935, 2010. 

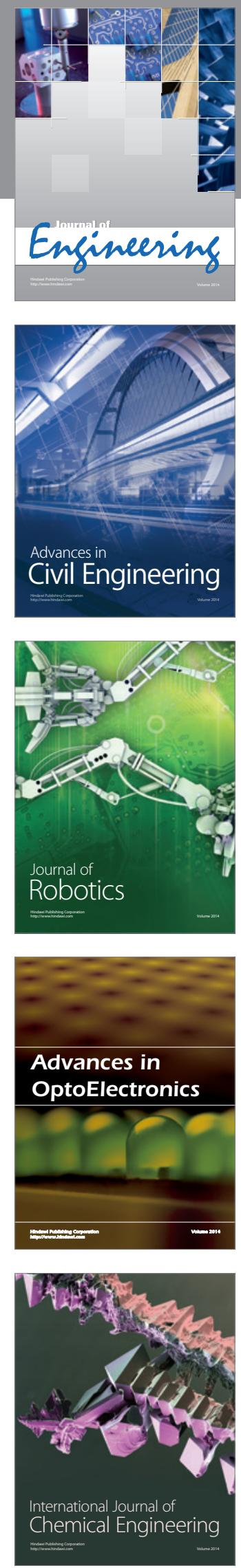

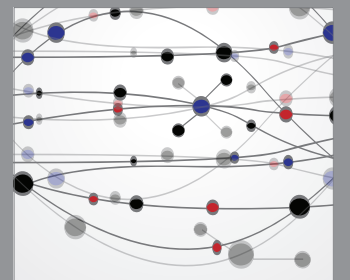

The Scientific World Journal
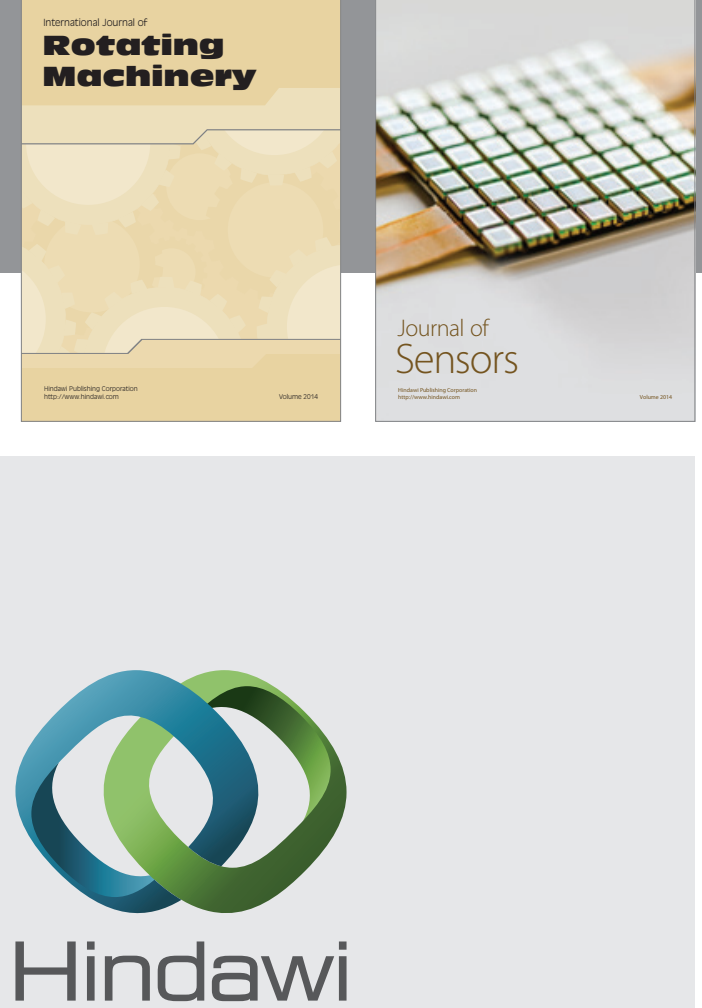

Submit your manuscripts at http://www.hindawi.com
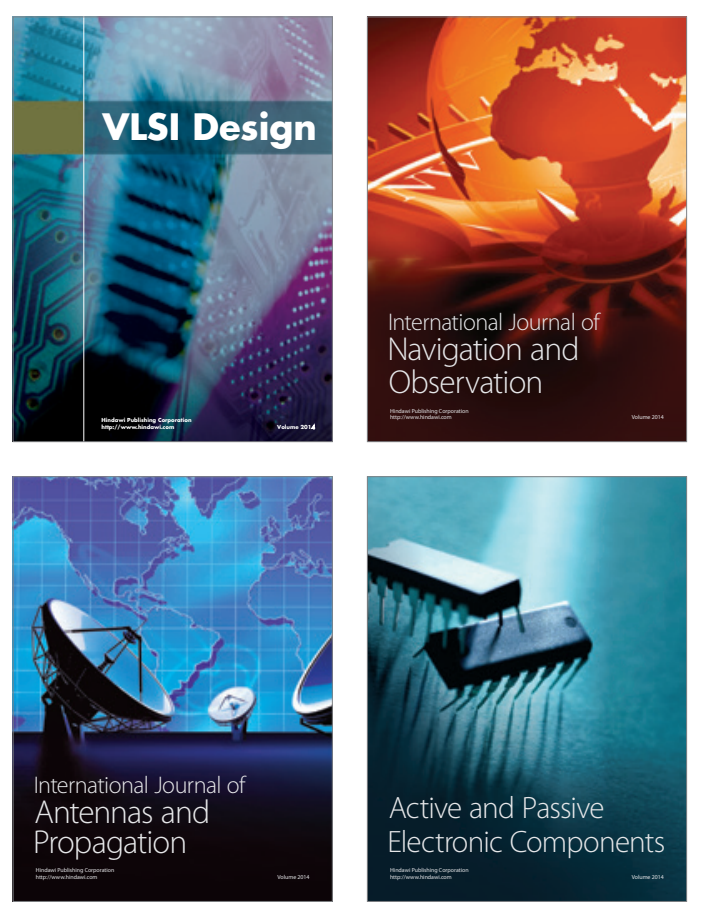
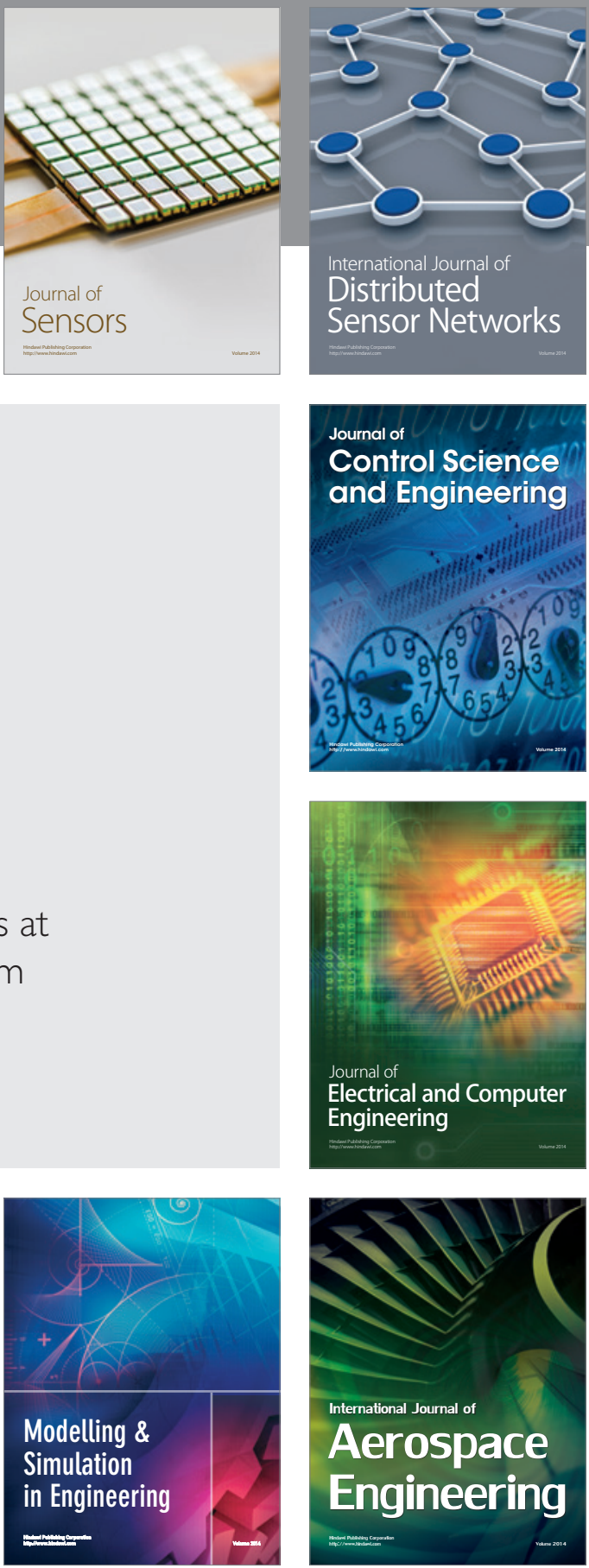

Journal of

Control Science

and Engineering
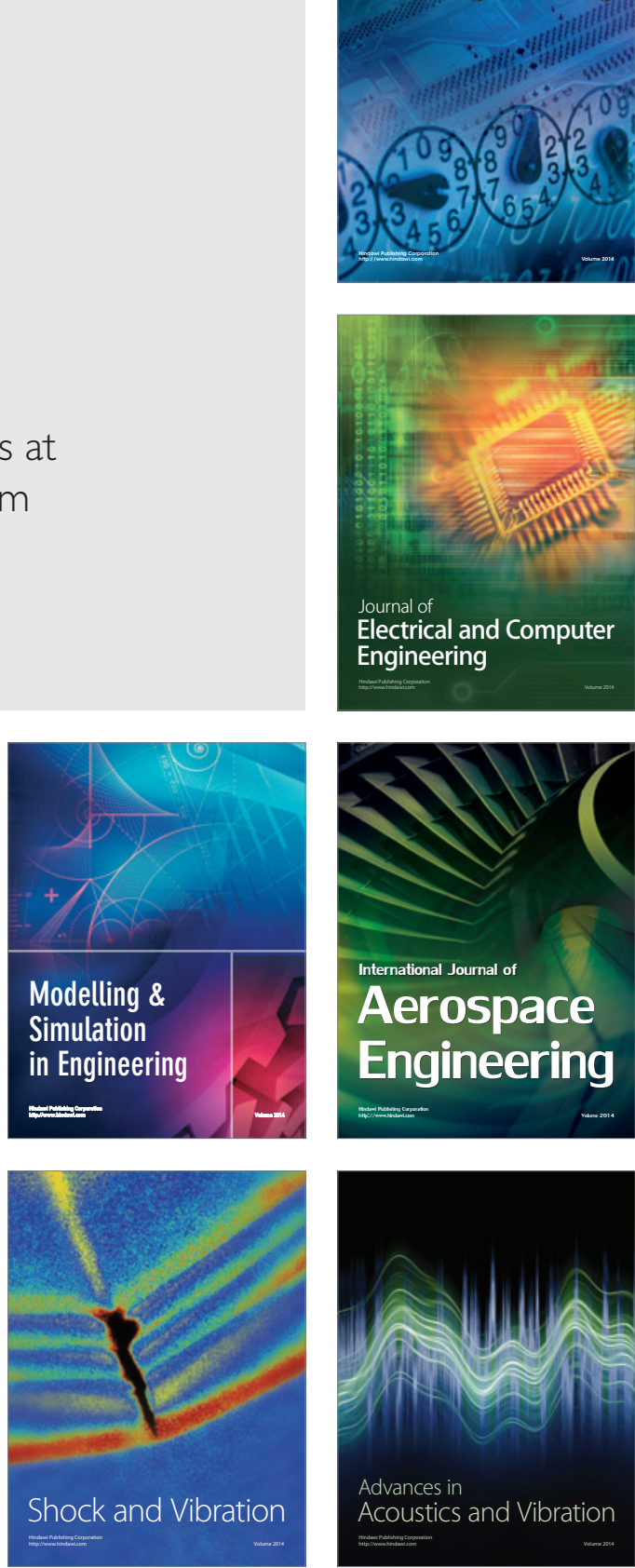